\title{
DYNAMIC FRACTURE EFFECTS ON EARTHQUAKES
}

\author{
Nobuki Yamagata ${ }^{1}$, Pedro V. Marcal $^{2}$ \\ ${ }^{1}$ Advanced Creative Technology Co., Ltd. Tokyo Japan (yamagata@actact.co.jp) \\ ${ }^{2}$ MPACT Corporation, CA USA (pedrovmarcal@gmail.com)
}

\begin{abstract}
Yamagata, N and Marcal, PV [1], studied the dynamic effects on Fracture by separating the behavior into its modal components. Previous work had concluded that the dynamic effect may be neglected in comparison to the static effect.[2]. In [1] the authors showed that by including the dynamic terms in the equivalent of a J-Integral Analysis, we obtain the surprising effect that the value of $J$ is a constant for each mode and it is equal to the maximum $J$ that can be calculated for that mode. In a sense this result supports the conclusion of [2]. However its implication in design has very serious unconservative consequences for failure particularly for earthquake and other design for dynamic impact. The traditional method of designing is based on the Square Root of the sum of the squares. This assumes an orthogonal combination of the modes. Because of the constant value of J for each mode, we need to design on the basis of the sum of the modes. This is a much less conservative approach.

In this paper, we give a case study of the dynamic effect on fracture by comparing the factors of safety for the current design procedures and the new procedure based on the results here.
\end{abstract}

Keywords: J-Integral, Fracture Mechanics, Crack, Finite Element Method, Eigen Mode.

\section{INTRODUCTION}

The application of fracture mechanics to static behavior is now well understood [3][4]. The equivalent critical measures are $\mathrm{K}$ the stress intensity factor, $\mathrm{J}$ the $\mathrm{J}$-integral and $\delta$ the crack opening displacement. The application of the metrics in design is circumscribed by wellknown factors such as modifications for elastic-plastic behavior, size effects etc.

The mechanics of a crack in the dynamic range can be divided into three parts. The first is the structural dynamic effect on the initiation and propagation of the crack. The second is the acoustic signature of the crack for purposes of detection. Finally the mechanics of the dynamic crack propagation. 
In this paper we will concentrate on the first behavior and concentrate on the macro behavior of the cracked structure. Apart from numerical simulations, there is little in the literature concerning crack initiation in the dynamic regime.

Budianski and Rice [5] considered the energy release of a crack from a conservation of energy point of view. In general it is surprising to see how the dynamic effects contained in the dynamic energy is ignored in the fracture process. Irwin [2] concluded that the dynamic effects were negligible when compared to the energy at the crack tip. Basically the J-Integral is evaluated around a path-independent line integral and by comparison the dynamic energy in a decreasing path was deemed to be negligible. Similarly in experimental verification in conjunction with numerical analysis [6], Weisbrod and Rittel used Stress Intensity Factors which implicitly neglects the dynamic effect except that which is included in the numerical analysis.

In this paper we follow the traditional analysis of Griffith[3] and Rice[4] but also include the term containing inertia to give us a starting point of Total Potential energy. We then calculate the Potential Energy in a structure and calculate its rate of change w.r.t. a crack length. The dynamic calculations are much simplified by adopting dynamic modal analysis. This approach also establishes the groundwork for its later application in earthquake analysis. Once the initial equations have been derived such an approach can be regarded as a postprocessing step to be added to the work performed to establish the initial static and modal analysis.

\section{THEORETICAL CONSIDERATIONS}

In this paper we reprise the equations developed in [1] in order to give the reader a full appreciation of the discussion and conclusions drawn here.

We define the total energy $\mathrm{E}$ in a finite element system in terms of its components:

$$
\mathrm{E}=-\mathrm{W}_{\mathrm{e}}+\mathrm{W}_{\mathrm{i}}+\mathrm{V}
$$

Where

$-\mathrm{W}_{\mathrm{e}}$, the external work,

$\mathrm{W}_{\mathrm{i}}$, inertial work using D'Alembert's principle

and $\mathrm{V}$, the internal strain energy

We note that the differential in the energy is the principle of Virtual work, i.e.

In equilibrium we have

$$
d E=-d W_{e}+d W_{i}+d V=0
$$

Now we introduce the effect of a crack extension da for a constant load 


$$
\mathrm{dE}_{\mathrm{c}}=-\mathrm{dW}_{\mathrm{e}}+\mathrm{dW}_{\mathrm{i}}+\mathrm{dV}+\partial \mathrm{W}_{\mathrm{i}} / \partial \mathrm{a} \mathrm{da}+\partial \mathrm{V} / \partial \mathrm{a} \mathrm{da}
$$

The energy absorbed by the crack is assumed to be constant.

Due to equilibrium in both the crack geometries a and a+da, the first three terms are equal to zero.

In the static case, introducing the usual FEA notation, we have

$$
\mathrm{dE}_{\mathrm{c}}=\mathrm{J} \text { da }=1 / 2 \mathrm{u}^{\mathrm{T}} \partial \mathrm{K} / \partial \mathrm{a} \mathrm{u} \mathrm{da}
$$

From which we obtain the J-Integral

$$
J=1 / 2 u^{T} \partial K / \partial a u
$$

In the dynamic case, assuming linearity, we have

$$
J=1 / 2 u^{T} \partial K / \partial a u+1 / 2 u^{T} \partial M / \partial a u
$$

where

$\mathrm{K}$ is the global stiffness

$\mathrm{u}$ is the vector of displacements

$\mathrm{M}$ is the Global Mass Matrix

We can see from the above that the dynamic term has the same structure as the stiffness terms and that there are no grounds for its neglect at this point. Following its name in the static case we will refer to the $\mathrm{J}$ defined in (5) as the dynamic J-Integral.

The addition of the word Integral is not strictly true in that the rate of the kinetic energy is not bounded by a path independent line integral as the strain energy rate is in the static case [2].

In the dynamic case it is convenient to continue the discussion in modal terms for a frequency $\omega_{\mathrm{i}}$ and retaining only the sine term,

$$
\begin{aligned}
\mathrm{dE}_{\mathrm{ci}}=\mathrm{J}_{\mathrm{i}} \mathrm{da}=1 / 2 \omega_{\mathrm{i}}^{2} & \cos ^{2} \omega_{\mathrm{i}} \mathrm{t} \cdot \mathrm{u}^{\mathrm{T}} \partial \mathrm{M} / \partial \mathrm{a} \mathrm{u} \mathrm{da} \\
& +1 / 2 \sin ^{2} \omega_{\mathrm{i}} \mathrm{t} \cdot \mathrm{u}^{\mathrm{T}} \partial \mathrm{K} / \partial \mathrm{a} \mathrm{u} \mathrm{da}
\end{aligned}
$$

and since in modal terms and using the eigenvalue equations,

$$
\mathrm{u}^{\mathrm{T}} \mathrm{Ku}=\omega_{\mathrm{i}}^{2} \mathrm{u}^{\mathrm{T}} \mathrm{Mu}
$$

we have for the dynamic case from (5)

$$
J_{i}=1 / 2 u_{i}^{T} \partial K / \partial a u_{i}
$$


This resulting equation is surprising in its simplicity. The conclusion is that the $\mathrm{J}$ integral is defined by the same equation in modal dynamics as in the static case providing that the displacement terms are a result of the appropriate dynamic analysis and for a particular mode. There is however one important difference in its implication and that is that the J-Integral remains constant throughout its cycle. This may be interpreted as the alternate conversion from static to dynamic analysis. Since the energy remains constant during the cycle, it should not be surprising that the J-Integral remains constant.

The total J-Integral for all the modes is obtained by a summation over all the modes,

$$
\begin{gathered}
\mathrm{J}=1 / 2 \sum \mathrm{u}_{\mathrm{i}}^{\mathrm{T}} \partial \mathrm{K} / \partial \mathbf{a} \mathrm{u}_{\mathrm{i}} \\
\text { for } \mathrm{i}=0 \text { to } \mathrm{n}
\end{gathered}
$$

With this explanation we can conclude that any evaluation using only the static $\mathbf{J}$ formula will result in an under-estimate of the fracture effect, also that any experimental determination neglecting the dynamic effects such as in [5] will result in a conservative fracture criteria.

\section{DESIGN CRITERIA}

In a normal seismic response analysis the combination of the modes flow naturally out of the observation that the modes are orthogonal. So we have the Square Root of the Sum of the Squares ( S.R.S.S.). However in light of the above, the criteria for the calculation of the JIntegral should be the Sum of the Modes (S.M.).

\section{CASE STUDY}

The calculation of the J-Integral form a seismic spectrum involves a Finite Element Analysis with an embedded crack and taking account of all the factors such as damping and soil behavior specified by the Codes.

In the shear wall analysis of [6] shown in Fig.1, We adopt the simplifying assumption that the J-Integral of each mode is proportional to the accelerating force given by the acceleration Response Spectra given in Fig. 2.

Then the J-Integral from S.R.S.S. is given by $\sqrt{ } 0.4 * 0.4+0.33 * 0.33+0.24 * 0.24=0.57$ And that from S.M. is 0.97 . The error in using S.R.S.S. is thus $(0.97-0.57) / 0.57 * 100=70 \%$.

This simple example shows the difference that can result from not using the correct Design Criteria for Dynamic Fracture Analysis. It is interesting to speculate that the recent Piping Failures due to Seismic events in Nuclear Plants may have resulted from the application of the S.R.S.S. to their design. 


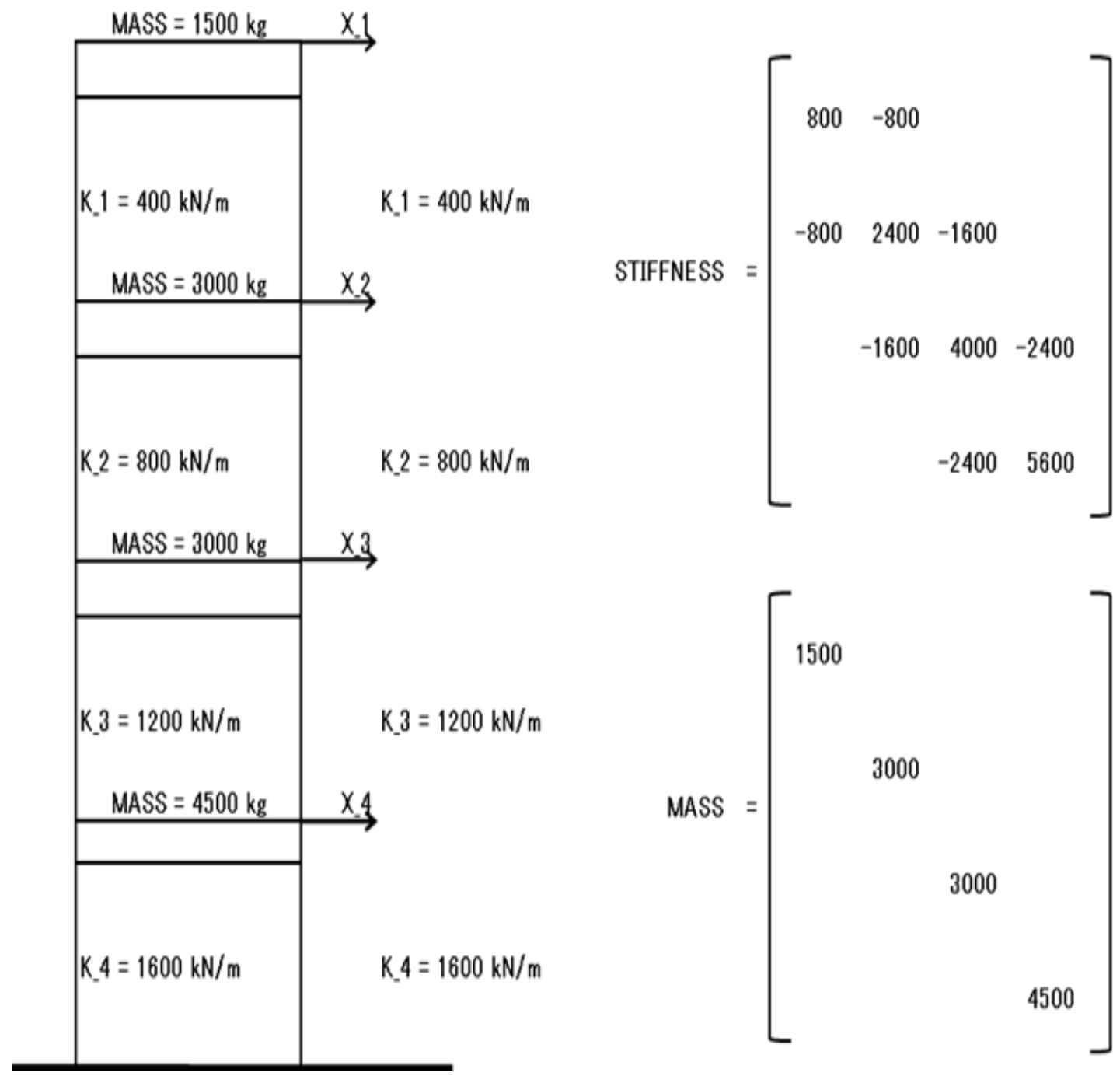

Fig. 1 Shear Building Model

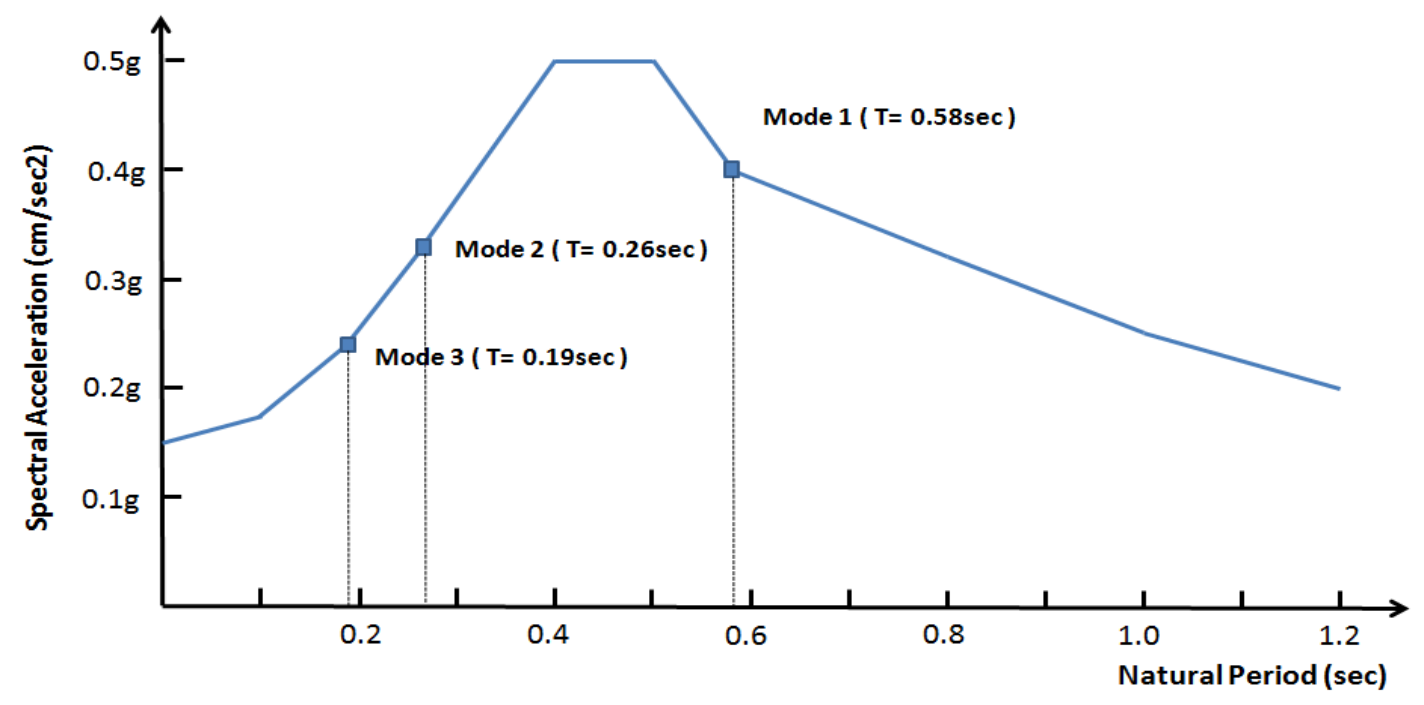

Fig. 2 Acceleration Response Spectra 


\section{CONCLUSIONS, RECOMMENDATIONS FOR FURTHER WORK}

We have demonstrated the need to include the dynamic effects in the evaluation of the $\mathrm{J}$ Integral modal response of a structure to a seismic event. We re-emphasize that this results in the J-Integral being constant throughout each mode. The combination of the modes should therefore follow a simple summation of the results for each mode (S.M.). In view of the progress being shown in geologic calculation of seismic effects [8], it will be interesting to use the calculations there to extract the value of the dynamic J-Integral for a typical seismic event.

\section{REFERENCES}

[1] Yamagata, N. and Marcal, P.V. "Structural Dynamic Analysis for Fracture", Proc. USNCCM-9, Los Angeles, 2007

[2] Irwin, G.R.,"Fracture Dynamics" Fracturing of Metals, American Society for Metals, Cleveland, 1948.

[3] Griffith, A.A., Phil Trans. Royal Soc., 1921 , Vol 221 , 163-198

[4] Rice, J.R. A path independent integral and the approximate analysis of strain concentration by notches and cracks. J. Appl. Mech. 35, $1968: 379-386$

[5] B. Budiansky and J. R. Rice, "Conservation Laws and Energy-Release Rates", Journal of Applied Mechanics, 40, 1973, pp. 201-203

[6] Weisbrod, G. and Rittel, D., "A method for dynamic fracture toughness determination using short beams", Int Journal of Fracture, 2000, 104, 89-103

[7] Wikipedia on Seismic Analysis, "Sample response example" , wikipedia,http://en.wikipedia.org/wiki/Seismic_analysis

[8] Dalguer, L. A. and Day, S. M., "Staggered-grid split-node method for spontaneous rupture simulation.” Jnl. Geophysical Res., Vol. 112, B02302, 2007 